\title{
A new non-linear limb-darkening law for LTE stellar atmosphere models II ${ }^{\star}$
}

\author{
Geneva and Walraven systems: Calculations for $-5.0 \leq \log [M / H] \leq+1$, \\ $2000 \mathrm{~K} \leq T_{\text {eff }} \leq 50000 \mathrm{~K}$ at several surface gravities
}

\begin{abstract}
A. Claret ${ }^{\star \star}$
Instituto de Astrofísica de Andalucía, CSIC, Apartado 3004, 18080 Granada, Spain

Received 3 December 2002 / Accepted 15 January 2003

Abstract. As an extension of our previous investigations on stellar atmospheres (Claret 2000), the limb-darkening coefficients for the Geneva and Walraven photometric systems are presented for the first time using the ATLAS and PHOENIX models. They cover a wide range of values of $\log g, T_{\text {eff }}$, metallicities and microturbulent velocities. In addition to the traditional applications of limb-darkening coefficients, the present ones are now being used in the interpretation of phase shift of B and A-F type pulsating stars.
\end{abstract}

Key words. stars: atmospheres - stars: binaries: eclipsing - stars: abundances

\section{Introduction}

In a previous paper (Claret 2000, Paper I) a new non-linear limb-darkening law was presented, that is capable of describe very accurately the specific intensity distribution. In addition, such a law can be applied in the whole HR diagram, instead of the bi-parametric laws which are barely adequate only in a certain range of effective temperatures and surface gravities. The corresponding non-linear limb-darkening coefficients were derived using the Least-Square Method (LSM) allowing a very good description of specific intensity $I(\mu)$ at any part of the disk, for any filter or wavelength, $\log g$, effective temperature, metallicity and microturbulent velocity. The results were tabulated for two of the most used photometric systems, uvby UBVRIJHK and bolometric and monochromatic calculations were also made available. The computations were presented for 19 metallicities ranging from $10^{-5}$ up to $10^{+1}$ solar abundances, $0 \leq \log g \leq 5$ and $2000 \mathrm{~K} \leq T_{\text {eff }} \leq 50000 \mathrm{~K}$. For the solar composition, we have also computed limb-darkening coefficients (LDC) for microturbulent velocities of $0,1,2$, $4,8 \mathrm{~km} \mathrm{~s}^{-1}$. It was also shown in that paper that the Flux Conservation Method (FCM) is not adequate to describe $I(\mu)$, mainly near the border of the disk, which are the most crucial points.

\footnotetext{
* Tables 2-38 are available in electronic form at the CDS via anonymous ftp to cdsarc.u-strasbg.fr $(130.79 .128 .5)$ or via http://cdsweb.u-strasbg.fr/cgi-bin/qcat?]/A+A/401/657. Additional data are available on CD ROMs upon request.

$\star \star$ e-mail: claret@iaa.es
}

Although the mentioned calculations cover the needs of several fields of astrophysics, many observations are now being carried out in less known photometric systems, such as the Geneva and Walraven ones. To give an example of a recent application, they are now being utilized in investigations on phase shifts of pulsating B and A-F stars (Daszynska 2002). The importance of the corresponding limb-darkening coefficients (LDC) for those stars and for other branches of stellar astrophysics are therefore obvious. The aim of this short paper is to present such calculations.

\section{The calculations and the table organization}

The importance of the LDC in astrophysics in general were discussed in detail in the previous papers and we do not repeat here the arguments; we only add to that list the mentioned research on the phase shift of pulsating B and A-F stars (Daszynska 2001, 2002). The numerical procedure and methodology which will be used here, as well as the input data, were described in detail in Paper I; the only difference is the filter transmission: Geneva system (Golay 1962) and Walraven (Walraven \& Walraven 1960). As in Paper I, we have used the ATLAS (Kurucz 2000) and PHOENIX (Haulschildt 2000) models. We have performed calculations of LDC for the same grids as before, that is, 19 metallicities ranging from $10^{-5}$ up to $10^{+1}$ solar abundances, $0 \leq \log g \leq 5$ and $2000 \mathrm{~K} \leq T_{\text {eff }} \leq 50000 \mathrm{~K}$ as well as for 5 values of the microturburlent velocities $(0,1,2$, 

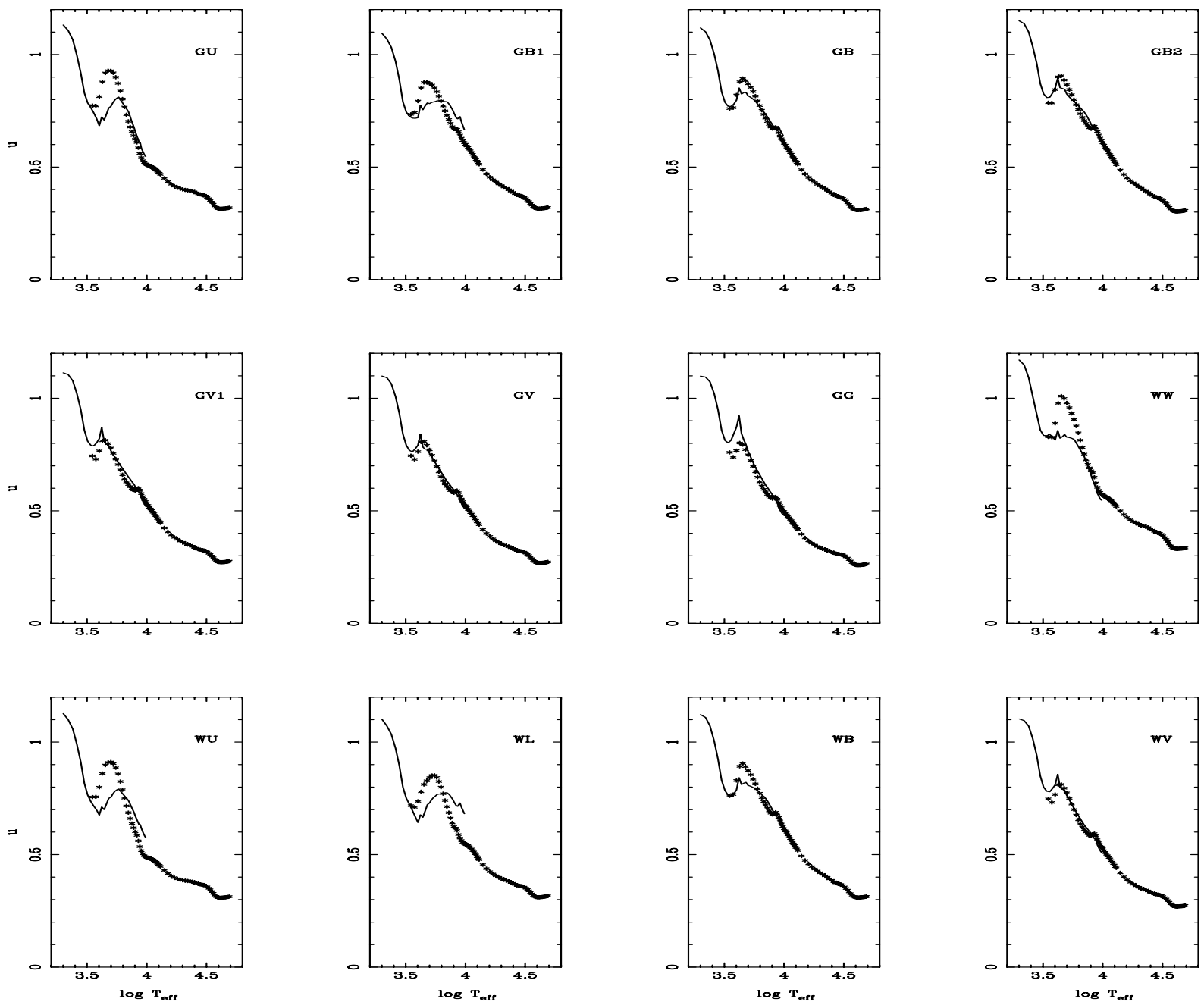

Fig. 1. The linear coefficient $u$ following PHOENIX models (continuous lines) and ATLAS (asterisks). $\log g=4.5$, solar abundance.

4, $8 \mathrm{~km} \mathrm{~s}^{-1}$ ). We reproduce below the adopted LDC laws in order to facilitate the identification of the coefficients contained in the tables:

Linear

$\frac{I(\mu)}{I(1)}=1-u(1-\mu)$

Quadratic

$\frac{I(\mu)}{I(1)}=1-a(1-\mu)-b(1-\mu)^{2}$

Square root

$\frac{I(\mu)}{I(1)}=1-c(1-\mu)-d(1-\sqrt{\mu})$

Logarithmic

$\frac{I(\mu)}{I(1)}=1-e(1-\mu)-f \mu \ln (\mu)$

where $I(1)$ is the specific intensity at the center of the disk, $u, a, b, c, d, e, f$ are the corresponding $\operatorname{LDC}$ and $\mu=\cos (\gamma)$, $\gamma$ being the angle between the line of sight and the emergent intensity. The proposed new law introduced in Paper I can be written as

$\frac{I(\mu)}{I(1)}=1-a_{1}\left(1-\mu^{1 / 2}\right)-a_{2}(1-\mu)-a_{3}\left(1-\mu^{3 / 2}\right)-a_{4}\left(1-\mu^{2}\right)$.

We shall denote the Geneva filters with a prefix $G$ in order to differentiate them from the corresponding filter in the Walraven system (prefix $W$ ) because there are some common letters to design the bands. In this way, the filters are denoted by $G U G B 1 G B G B 2 G V 1 G V G G W W W U W L W B W V$. As commented, we have adopted the LSM, which is superior to the FCM and we refer the interested readers to Paper I for a detailed comparison. This superiority was again detected in this series of calculations whatever the filter, effective temperature, $\log g$, metallicity and microturburlent velocity. The superiority of the LSM over FCM is clear whatever the law used. Among other reasons (see Sects. 2.2 and 3.1 in Paper I), the FCM method does not use any kind of information on how the intensity is distributed over the disk and an extra condition (arbitrary) to derive the LDC is, therefore, necessary (the arbitrariness increasing with the complexity of the corresponding law). 

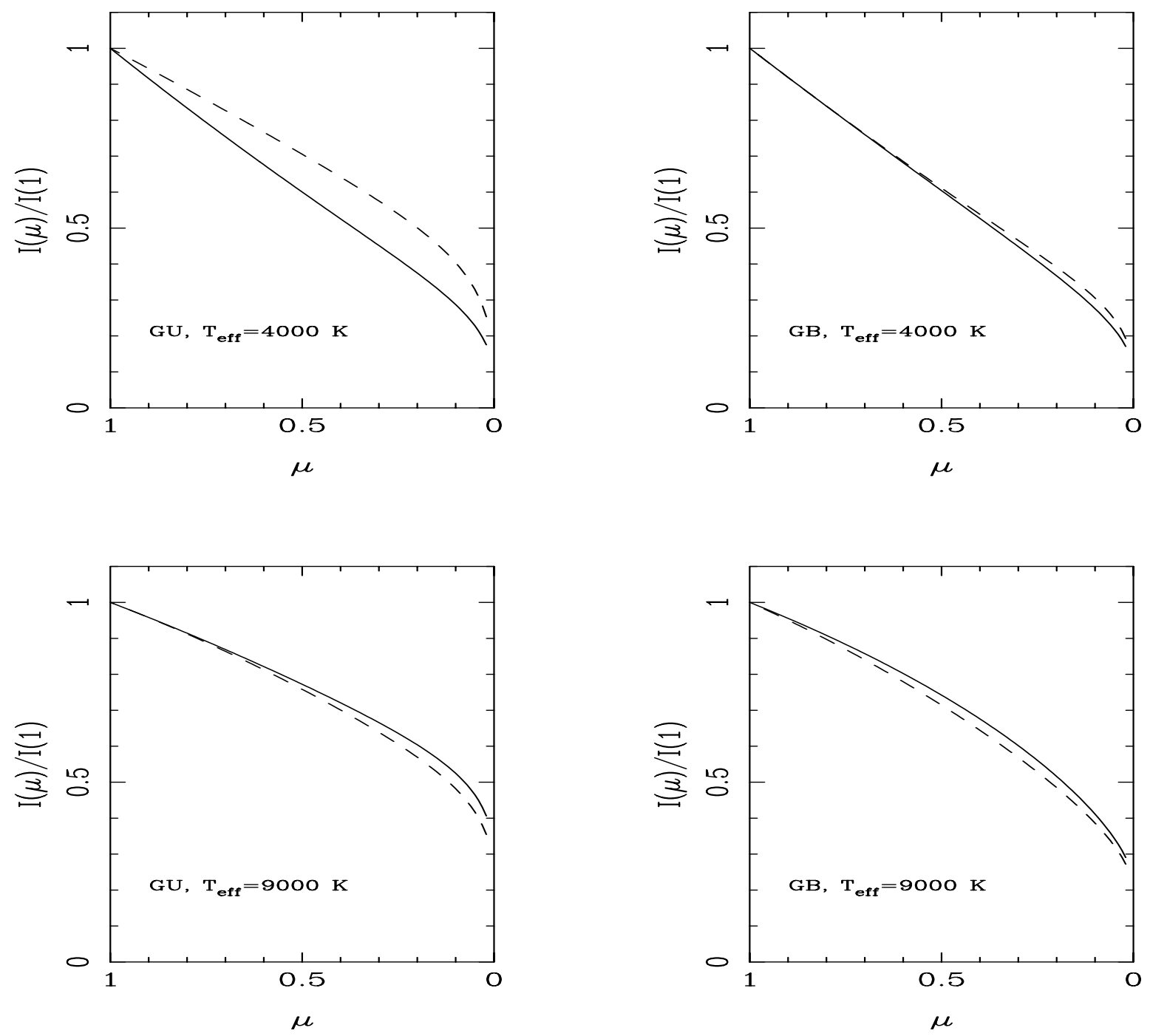

Fig. 2. The specific intensities as a function of $\mu$ for $T_{\text {eff }}=4000 \mathrm{~K}$ and $9000 \mathrm{~K}(\log g=4.5)$. ATLAS models are represented by full lines while dashed ones denote PHOENIX models. Solar metallicity.

As an example of the behavior of the derived LDC we can see in Fig. 1 how the coefficient $u$ depends on the effective temperature for ATLAS and PHOENIX models. For $6300 \mathrm{~K} \leq T_{\text {eff }} \leq 9800 \mathrm{~K}$ and for larger passbands the comparison shows an acceptable agreement while for smaller $T_{\text {eff }}$ the interagreement is worse. This comparison presents similarities with the one discussed in Claret (1998) when comparing the ATLAS and PHOENIX models in the LDC calculations for uvby $U B V R I J H K$ filters. Figure 2 shows the intensities for two models $\left(T_{\mathrm{eff}}=4000 \mathrm{~K}\right.$ and $9000 \mathrm{~K}, \log g=4.5$, filters $G U$ and $G B$ ) using ATLAS (full line) and PHOENIX (dashed one). The new non-linear law was adopted. These models confirm the aspect suggested by Fig. 1: for lower effective temperatures and small effective wavelength, a large difference is detected. Note that such a disagreement is due to the input physics of both codes. We do not reproduce the arguments in Paper I and in Claret (1998). We refer the interested readers to both papers. However, some words of caution on this kind of comparison are needed. Although the number of $\mu$ points are similar in ATLAS and PHOENIX codes, their distribution are not the same. This has immediate implication for the interpretation of, for example, bi-parametric coefficients. So, the differences shown in Figs. 1 and 2 cannot be attributed only to differences in the physics of the codes but also to the angular distribution of points.

Finally, some words concerning the table organization. Table 1 summarizes the main characteristics of the computed LDC and it is self explanatory. We encourage the reader to use the new non-linear law since it provides a very good description of $I(\mu)$ (with merit functions about $10^{3}-10^{4}$ times smaller than those for linear and bi-parametric approximations (see Paper I). However, if one is interested in using the linear or bi-parametric approximations, additional tables containing the respectives merit functions are also available to evaluate the errors. Although the FCM does not provide good fits to the actual specific intensities, the LDC based on this method are also available on request. The same holds for the monochromatic and actual intensities for the 12 passbands.

Acknowledgements. R. Kurucz and P. H. Hauschildt are acknowledged for providing the data of their respective atmosphere models. The Spanish DGYCIT (PB98-0499) is gratefully acknowledged for its support during the development of this work. 
Table 1. Limb-Darkening coefficients for $G U G B 1 G B G B 2 G V 1 G V G G W W W U W L W B W V$ bands (Least-Squares Method).

\begin{tabular}{|c|c|c|c|c|c|c|}
\hline Name & Source & $\overline{\text { range } T_{\text {eff }}}$ & range $\log g$ & $\log [\mathrm{M} / \mathrm{H}]$ & Vel Turb. & Kind of fitting \\
\hline Table 2 & ATLAS & $3500 \mathrm{~K}-50000 \mathrm{~K}$ & $0.0-5.0$ & -5.0 & $2 \mathrm{~km} \mathrm{~s}^{-1}$ & Eq. (5), 12 filters \\
\hline Table 3 & ATLAS & $3500 \mathrm{~K}-50000 \mathrm{~K}$ & $0.0-5.0$ & -4.5 & $2 \mathrm{~km} \mathrm{~s}^{-1}$ & Eq. (5), 12 filters \\
\hline Table 4 & ATLAS & $3500 \mathrm{~K}-50000 \mathrm{~K}$ & $0.0-5.0$ & -4.0 & $2 \mathrm{~km} \mathrm{~s}^{-1}$ & Eq. (5), 12 filters \\
\hline Table 5 & ATLAS & $3500 \mathrm{~K}-50000 \mathrm{~K}$ & $0.0-5.0$ & -3.5 & $2 \mathrm{~km} \mathrm{~s}^{-1}$ & Eq. (5), 12 filters \\
\hline Table 6 & ATLAS & $3500 \mathrm{~K}-50000 \mathrm{~K}$ & $0.0-5.0$ & -3.0 & $2 \mathrm{~km} \mathrm{~s}^{-1}$ & Eq. (5), 12 filters \\
\hline Table 7 & ATLAS & $3500 \mathrm{~K}-50000 \mathrm{~K}$ & $0.0-5.0$ & -2.5 & $2 \mathrm{~km} \mathrm{~s}^{-1}$ & Eq. (5), 12 filters \\
\hline Table 8 & ATLAS & $3500 \mathrm{~K}-50000 \mathrm{~K}$ & $0.0-5.0$ & -2.0 & $2 \mathrm{~km} \mathrm{~s}^{-1}$ & Eq. (5), 12 filters \\
\hline Table 9 & ATLAS & $3500 \mathrm{~K}-47500 \mathrm{~K}$ & $0.0-5.0$ & -1.5 & $2 \mathrm{~km} \mathrm{~s}^{-1}$ & Eq. (5), 12 filters \\
\hline Table 10 & ATLAS & $3500 \mathrm{~K}-50000 \mathrm{~K}$ & $0.0-5.0$ & -1.0 & $2 \mathrm{~km} \mathrm{~s}^{-1}$ & Eq. (5), 12 filters \\
\hline Table 11 & ATLAS & $3500 \mathrm{~K}-50000 \mathrm{~K}$ & $0.0-5.0$ & -0.5 & $2 \mathrm{~km} \mathrm{~s}^{-1}$ & Eq. (5), 12 filters \\
\hline Table 12 & ATLAS & $3500 \mathrm{~K}-50000 \mathrm{~K}$ & $0.0-5.0$ & -0.3 & $2 \mathrm{~km} \mathrm{~s}^{-1}$ & Eq. (5), 12 filters \\
\hline Table 13 & ATLAS & $3500 \mathrm{~K}-50000 \mathrm{~K}$ & $0.0-5.0$ & -0.2 & $2 \mathrm{~km} \mathrm{~s}^{-1}$ & Eq. (5), 12 filters \\
\hline Table 14 & ATLAS & $3500 \mathrm{~K}-50000 \mathrm{~K}$ & $0.0-5.0$ & -0.1 & $2 \mathrm{~km} \mathrm{~s}^{-1}$ & Eq. (5), 12 filters \\
\hline Table 15 & ATLAS & $3500 \mathrm{~K}-50000 \mathrm{~K}$ & $0.0-5.0$ & 0.0 & $2 \mathrm{~km} \mathrm{~s}^{-1}$ & Eq. (5), 12 filters \\
\hline Table 16 & ATLAS & $3500 \mathrm{~K}-50000 \mathrm{~K}$ & $0.0-5.0$ & +0.1 & $2 \mathrm{~km} \mathrm{~s}^{-1}$ & Eq. (5), 12 filters \\
\hline Table 17 & ATLAS & $3500 \mathrm{~K}-50000 \mathrm{~K}$ & $0.0-5.0$ & +0.2 & $2 \mathrm{~km} \mathrm{~s}^{-1}$ & Eq. (5), 12 filters \\
\hline Table 18 & ATLAS & $3500 \mathrm{~K}-50000 \mathrm{~K}$ & $0.0-5.0$ & +0.3 & $2 \mathrm{~km} \mathrm{~s}^{-1}$ & Eq. (5), 12 filters \\
\hline Table 19 & ATLAS & $3500 \mathrm{~K}-45000 \mathrm{~K}$ & $0.0-5.0$ & +0.5 & $2 \mathrm{~km} \mathrm{~s}^{-1}$ & Eq. (5), 12 filters \\
\hline Table 20 & ATLAS & $3500 \mathrm{~K}-40000 \mathrm{~K}$ & $0.0-5.0$ & +1.0 & $2 \mathrm{~km} \mathrm{~s}^{-1}$ & Eq. (5), 12 filters \\
\hline Table 21 & ATLAS & $3500 \mathrm{~K}-50000 \mathrm{~K}$ & $0.0-5.0$ & 0.0 & $0 \mathrm{~km} \mathrm{~s}^{-1}$ & Eq. (5), 12 filters \\
\hline Table 22 & ATLAS & $3500 \mathrm{~K}-50000 \mathrm{~K}$ & $0.0-5.0$ & 0.0 & $1 \mathrm{~km} \mathrm{~s}^{-1}$ & Eq. (5), 12 filters \\
\hline Table 23 & ATLAS & $3500 \mathrm{~K}-50000 \mathrm{~K}$ & $0.0-5.0$ & 0.0 & $4 \mathrm{~km} \mathrm{~s}^{-1}$ & Eq. (5), 12 filters \\
\hline Table 24 & ATLAS & $3500 \mathrm{~K}-50000 \mathrm{~K}$ & $0.0-5.0$ & 0.0 & $8 \mathrm{~km} \mathrm{~s}^{-1}$ & Eq. (5), 12 filters \\
\hline Table 25 (Sun) & ATLAS & $5777 \mathrm{~K}$ & 4.377 & 0.0 & $1.5 \mathrm{~km} \mathrm{~s}^{-1}$ & Eq. (5), 12 filters \\
\hline Table 26 (Vega) & ATLAS & $9400 \mathrm{~K}$ & 3.90 & -0.5 & $0 \mathrm{~km} \mathrm{~s}^{-1}$ & Eq. (5), 12 filters \\
\hline Table 30 & ATLAS & $3500 \mathrm{~K}-50000 \mathrm{~K}$ & $0.0-5.0$ & all metallicities & $0,1,2,4,8 \mathrm{~km} \mathrm{~s}^{-1}$ & Eq. (1), 12 filters \\
\hline Table 31 & ATLAS & $3500 \mathrm{~K}-50000 \mathrm{~K}$ & $0.0-5.0$ & all metallicities & $0,1,2,4,8 \mathrm{~km} \mathrm{~s}^{-1}$ & Eq. (2), 12 filters \\
\hline Table 32 & ATLAS & $3500 \mathrm{~K}-50000 \mathrm{~K}$ & $0.0-5.0$ & all metallicities & $0,1,2,4,8 \mathrm{~km} \mathrm{~s}^{-1}$ & Eq. (3), 12 filters \\
\hline Table 33 & ATLAS & $3500 \mathrm{~K}-50000 \mathrm{~K}$ & $0.0-5.0$ & all metallicities & $0,1,2,4,8 \mathrm{~km} \mathrm{~s}^{-1}$ & Eq. (4), 12 filters \\
\hline Table 34 & PHOENIX & $2000 \mathrm{~K}-9800 \mathrm{~K}$ & $3.5-5.0$ & 0 & $2 \mathrm{~km} \mathrm{~s}^{-1}$ & Eq. (5), 12 filters \\
\hline Table 35 & PHOENIX & $2000 \mathrm{~K}-9800 \mathrm{~K}$ & $3.5-5.0$ & 0 & $2 \mathrm{~km} \mathrm{~s}^{-1}$ & Eq. (1), 12 filters \\
\hline Table 36 & PHOENIX & $2000 \mathrm{~K}-9800 \mathrm{~K}$ & $3.5-5.0$ & 0 & $2 \mathrm{~km} \mathrm{~s}^{-1}$ & Eq. (2), 12 filters \\
\hline Table 37 & PHOENIX & $2000 \mathrm{~K}-9800 \mathrm{~K}$ & $3.5-5.0$ & 0 & $2 \mathrm{~km} \mathrm{~s}^{-1}$ & Eq. (3), 12 filters \\
\hline Table 38 & PHOENIX & $2000 \mathrm{~K}-9800 \mathrm{~K}$ & $3.5-5.0$ & 0 & $2 \mathrm{~km} \mathrm{~s}^{-1}$ & Eq. (4), 12 filters \\
\hline
\end{tabular}

\section{References}

Claret, A. 1998, A\&A, 335, 647

Claret, A. 2000, A\&A, 363, 1081

Daszynska, J. 2001, Ph.D. Thesis, Wroclaw University

Daszynska, J. 2002, private communication
Golay, M. 1972, Vistas Astron., 14, 13

Hauschildt, P. H. 2000, private communication

Kurucz, R. L. 2000, private communication

Walraven, T., \& Walraven, J. H. 1960, BAN, 15, 67 